International Journal of Pure and Applied Mathematics

Volume 108 No. $4 \quad$ 2016, 921-928

ISSN: 1311-8080 (printed version); ISSN: 1314-3395 (on-line version)

url: http://www.ijpam.eu

doi: 10.12732 /ijpam.v108i4.17

\title{
NONHOLONOMIC FRAMES FOR FINSLER SPACE WITH GENERALIZED KROPINA METRIC
}

\author{
Brijesh Kumar Tripathi ${ }^{1}$, V.K. Chaubey $^{2}$, R.B. Tiwari ${ }^{3} \S$ \\ ${ }^{1}$ Department of Mathematics \\ L.E. College \\ Morbi, Samakathe Morbi-2, (Gujrat), 363642, INDIA \\ ${ }^{2}$ Department of Applied Sciences \\ Buddha Institute of Technology, GIDA \\ Gorakhpur, U.P., 273209, INDIA \\ ${ }^{3}$ Department of Mathematics \\ M.K.R.E.C.I.T., Ambedkar Nagar U.P., INDIA
}

\begin{abstract}
The purpose of present paper to determine the Finsler spaces due to deformation of generalized Kropina metric. Consequently, we obtain the non-holonomic frame for generalized Kropina metric with condition $n \geq 1$, such as $L(\alpha, \beta)=\frac{\beta^{(n+1)}}{\alpha^{n}}$.
\end{abstract}

AMS Subject Classification: $53 \mathrm{C} 60$

Key Words: Finsler space with $(\alpha, \beta)$-metrics, generalized Kropina metric, GL-metric, nonholonomic Finsler frame

\section{Introduction}

In 1982, P.R. Holland [1], [2], studies a unified formalism that uses a nonholonomic frame on space time arising from consideration of a charged particle moving in an external electromagnetic field. In fact, R.S. Ingarden [3] was the first to point out that the Lorentz force law can be written in this case as geodesic equation on a Finsler space called Randers space. The author R.G. Beil [5], [6], have studied a gauge transformation viewed as a nonholonomic frame

Received: April 30, 2016

Published: August 16, 2016

$\S$ Correspondence author (c) 2016 Academic Publications, Ltd.

url: www.acadpubl.eu 
on the tangent bundle of a four dimensional base manifold. The geometry that follows from these considerations gives a unified approach to gravitation and gauge symmetries. In the present paper we have used the common Finsler idea to study the existence of a nonholonomic frame on the vertical sub bundle VTM of the tangent bundle of a base manifold $\mathrm{M}$.

In the present paper, the fundamental tensor field might be taught as the result of two Finsler deformations. Then we can determine a corresponding frame for each of these two Finsler deformations. Consequently, a nonholonomic frame for a Finsler space with Generalized Kropina Metric and discuss two special cases when $n=1$ and $n=2$. This is an extension work of Ioan Bucataru and Radu Miron [10], Tripathi [14] and Narasimhamurthy [15].

Consider, $a_{i j}(x)$ the components of a Riemannian metric on the base manifold M, $a(x, y)>0$ and $b(x, y) \geq 0$ Two functions on TM and $B(x, y)=$ $B_{i}(x, y)\left(d x^{i}\right)$ a vertical 1-form on TM. Then

$$
g_{i j}(x, y)=a(x, y) a_{i j}(x)+b(x, y) B_{i}(x, y) B_{j}(x, y)
$$

is a generalized Lagrange metric, called the Beil metric. The metric tensor $g_{i j}$ is also known as a Beil deformation of the Riemannian metric $a_{i j}$. It has been studied and applied by R. Miron and R.K. Tavakol in General Relativity for $a(x, y)=\exp (2 \sigma(x, y))$ and $\mathrm{b}=0$. The case $\mathrm{a}(\mathrm{x}, \mathrm{y})=1$ with various choices of $\mathrm{b}$ and $B_{i}$ was introduced and studied by R.G. Beil for constructing a new unified field theory [6].

\section{Preliminaries}

An important class of Finsler spaces is the class of Finsler spaces with $(\alpha, \beta)$ metrics [11]. The first Finsler spaces with $(\alpha, \beta)$-metrics were introduced by the physicist G. Randers in 1940, are called Randers spaces [4]. Recently, R.G. Beil suggested a more general case and considered the class of Lagrange spaces with $(\alpha, \beta)$-metric, which was discussed in [12]. A unified formalism which uses a nonholonomic frame on space time, a sort of plastic deformation, arising from consideration of a charged particle moving in an external electromagnetic field in the background space time viewed as a strained mechanism studied by P. R. Holland [1], [2]. If we do not ask for the function $\mathrm{L}$ to be homogeneous of order two with respect to the $(\alpha, \beta)$ variables, then we have a Lagrange space with $(\alpha, \beta)$-metric. Next we defined some different Finsler space with $(\alpha, \beta)$-metrics.

Definition 2.1. A Finsler space $F^{n}=\{M, F(x, y)\}$ is called with $(\alpha, \beta)$ metric if there exists a 2-homogeneous function $\mathrm{L}$ of two variables such that the 
Finsler metric $F: T M \rightarrow R$ is given by

$$
F^{2}(x, y)=L\{\alpha(x, y), \beta(x, y)\}
$$

where $\alpha^{2}(x, y)=a_{i j}(x) y^{i} y^{j}, \alpha$ is a Riemannian metric on the manifold M, and $\beta(x, y)=b_{i}(x) y^{i}$ is a 1 -form on $\mathrm{M}$

Consider $g_{i j}=\frac{1}{2} \frac{\partial^{2} F^{2}}{\partial y^{i} \partial y^{j}}$ the fundamental tensor of the Randers space(M,F). Taking into account the homogeneity of a and $\mathrm{F}$ we have the following formulae:

$$
\begin{gathered}
p^{i}=\frac{1}{a} y^{i}=a^{i j} \frac{\partial \alpha}{\partial y^{j}} ; \quad p_{i}=a_{i j} p^{j}=\frac{\partial \alpha}{\partial y^{i}} \\
l^{i}=\frac{1}{L} y^{i}=g^{i j} \frac{\partial l}{\partial y^{i}} ; l_{i}=g_{i j} l^{j}=\frac{\partial L}{\partial y^{i}}=P_{i}+b_{i} \\
l^{i}=\frac{1}{L} p^{i} ; l^{i} l_{i}=p^{i} p_{i}=1 ; l^{i} p_{i}=\frac{\alpha}{L} ; p^{i} l_{i}=\frac{L}{\alpha} \\
b_{i} P^{i}=\frac{\beta}{\alpha} ; b_{i} l^{i}=\frac{\beta}{L}
\end{gathered}
$$

with respect to these notations, the metric tensors $a_{i j}$ and $g_{i j}$ are related by [13],

$$
g_{i j}(x, y)=\frac{L}{\alpha} a_{i j}+b_{i} P_{j}+P_{i} b_{j}-\frac{\beta}{\alpha} p_{i} p_{j}=\frac{L}{\alpha}\left(a_{i j}-p_{i} p_{j}\right)+l_{i} l_{j}
$$

Theorem 2.1. [10]: For a Finsler space (M,F) consider the matrix with the entries:

$$
Y_{j}^{i}=\sqrt{\frac{\alpha}{L}}\left(\delta_{j}^{i}-l^{i} l_{j}+\sqrt{\frac{\alpha}{L}} p^{i} p_{j}\right)
$$

defined on TM. Then $Y_{j}=Y_{j}^{i}\left(\frac{\partial}{\partial y^{i}}\right), \quad j \in 1,2,3, \ldots, n$ is a non holonomic frame.

Theorem 2.2. [7]: With respect to frame the holonomic components of the Finsler metric tensor $a_{\alpha \beta}$ is the Randers metric $g_{i j}$, i.e,

$$
g_{i j}=Y_{i}^{\alpha} Y_{j}^{\beta} a_{\alpha \beta}
$$

Throughout this section we shall rise and lower indices only with the Riemannian metric $a_{i j}(x)$ that is $y_{i}=a_{i j} y^{j}, \beta^{i}=a^{i j} b_{j}$, and so on. For a Finsler space with $(\alpha, \beta)$-metric $F^{2}(x, y)=L\{\alpha(x, y), \beta(x, y)\}$ we have the Finsler invariants [13].

$$
\rho_{1}=\frac{1}{2 \alpha} \frac{\partial L}{\partial \alpha} ; \rho_{0}=\frac{1}{2} \frac{\partial^{2} L}{\partial \beta^{2}} ; \rho_{-1}=\frac{1}{2 \alpha} \frac{\partial^{2} L}{\partial \alpha \partial \beta} ; \rho_{-2}=\frac{1}{2 \alpha^{2}}\left(\frac{\partial^{2} L}{\partial \alpha^{2}}-\frac{1}{\alpha} \frac{\partial L}{\partial \alpha}\right)
$$


where subscripts $1,0,-1,-2$ gives us the degree of homogeneity of these invariants.

For a Finsler space with $(\alpha, \beta)$-metric we have,

$$
\rho_{-1} \beta+\rho_{-2} \alpha^{2}=0
$$

with respect to the notations we have that the metric tensor $g_{i j}$ of a Finsler space with $(\alpha, \beta)$-metric is given by [13].

$$
g_{i j}(x, y)=\rho a_{i j}(x)+\rho_{0} b_{i}(x)+\rho_{-1}\left\{b_{i}(x) y_{j}+b_{j}(x) y_{i}\right\}+\rho_{-2} y_{i} y_{j}
$$

From (2.8) we can see that $g_{i j}$ is the result of two Finsler deformations:

$$
\begin{aligned}
\text { I. } & a_{i j} \rightarrow h_{i j}=\rho a_{i j}+\frac{1}{\rho_{-2}}\left(\rho_{-1} b_{i}+\rho_{-2} y_{i}\right)\left(\rho_{-1} b_{j}+\rho_{-2} y_{j}\right) \\
\text { II. } & h_{i j} \rightarrow g_{i j}=h_{i j}+\frac{1}{\rho_{-2}}\left(\rho_{0} \rho_{-1}-\rho_{-1}^{2}\right) b_{i} b_{j}
\end{aligned}
$$

The nonholonomic Finsler frame that corresponding to the $I^{s t}$ deformation (2.9) is according to the theorem (7.9.1) in [10], given by,

$$
X_{j}^{i}=\sqrt{\rho} \delta_{j}^{i}-\frac{1}{\beta^{2}}\left\{\sqrt{\rho}+\sqrt{\rho+\frac{\beta^{2}}{\rho_{-2}}}\right\}\left(\rho_{-1} b^{i}+\rho_{-2} y^{i}\right)\left(\rho_{-1} b_{j}+\rho_{-2} y_{j}\right)
$$

where $B^{2}=a_{i j}\left(\rho_{-1} b^{i}+\rho_{-2} y^{i}\right)\left(\rho_{-1} b_{j}+\rho_{-2} y_{j}\right)=\rho_{-1}^{2} b^{2}+\beta \rho_{-1} \rho_{-2}$.

This metric tensor $a_{i j}$ and $h_{i j}$ are related by,

$$
h_{i j}=X_{i}^{k} X_{j}^{l} a_{k l}
$$

Again the frame that corresponds to the $I I_{n d}$ deformation (2.9) given by,

$$
Y_{j}^{i}=\delta_{j}^{i}-\frac{1}{C^{2}}\left\{1 \pm \sqrt{1+\left(\frac{\rho_{-2} C^{2}}{\rho_{0} \rho_{-2}-\rho_{-1}^{2}}\right)}\right\} b^{i} b_{j}
$$

where $C^{2}=h_{i j} b^{i} b^{j}=\rho b^{2}+\frac{1}{\rho_{-2}}\left(\rho_{-1} b^{2}+\rho_{-2} \beta\right)^{2}$.

The metric tensor $h_{i j}$ and $g_{i j}$ are related by the formula;

$$
g_{m n}=Y_{m}^{i} Y_{n}^{j} h_{i j}
$$

Theorem 2.3. [10]: Let $F^{2}(x, y)=L\{\alpha(x, y), \beta(x, y)\}$ be the metric function of a Finsler space with $(\alpha, \beta)$ metric for which the condition (2.7) is true. Then

$$
V_{j}^{i}=X_{k}^{i} Y_{j}^{k}
$$

is a nonholonomic Finsler frame with $X_{k}^{i}$ and $Y_{j}^{k}$ are given by (2.10) and (2.12) respectively. 


\section{Nonholonmic Frames for Finsler Space with Generalized Kropina Metric}

In this section we consider the case of nonholonomic Finlser frame with Generalized Kropina metric $L(\alpha, \beta)=\frac{\beta^{2 n+2}}{\alpha^{2 n}}$

$$
\begin{array}{r}
\rho_{1}=-\frac{n \beta^{2 n+2}}{\alpha^{2 n+2}}, \quad \rho_{0}=\frac{(n+1)(2 n+1) \beta^{2 n}}{\alpha^{2 n}}, \\
\rho_{-1}=-\frac{2 n(n+1) \beta^{2 n+1}}{\alpha^{2 n+2}}, \quad \rho_{-2}=\frac{2 n(n+1) \beta^{2 n+2}}{\alpha^{2 n+4}}, \\
B^{2}=\frac{4 n^{2}(n+1)^{2} \beta^{4 n+2}\left(\alpha^{2} b^{2}-\beta^{2}\right)}{\alpha^{(4 n+6)}}
\end{array}
$$

Using (3.1) in (2.10) we have,

$$
\begin{aligned}
& X_{j}^{i}=\sqrt{-n} \frac{\beta^{n+1}}{\alpha^{n+1}} \delta_{j}^{i}-4 n^{2}(n+1)^{2} \frac{\beta^{4 n}}{\alpha^{4 n+4}}\left\{\sqrt{-n} \frac{\beta^{n+1}}{\alpha^{n+1}}+\right. \\
& \left.\sqrt{\left(-n \frac{\beta^{2 n+2}}{\alpha^{2 n+2}}+\frac{\alpha^{2 n+4}}{2 n(n+1) \beta^{2 n}}\right)}\right\}\left(b^{i}-\frac{\beta}{\alpha^{2}} y^{i}\right)\left(b_{j}-\frac{\beta}{\alpha^{2}} y_{j}\right)
\end{aligned}
$$

Again using (3.1) in (2.12) we have,

$$
Y_{j}^{i}=\delta_{j}^{i}-\frac{1}{C^{2}}\left\{1 \pm \sqrt{1+\frac{\alpha^{2 n} C^{2}}{(n+1) \beta^{2 n}}}\right\} b^{i} b_{j}
$$

where $C^{2}=-\frac{n \beta^{2 n+2}}{\alpha^{2 n+2}} b^{2}+\frac{2 n(n+1) \beta^{2 n}}{\alpha^{2 n+4}}\left(\alpha^{2} b^{2}-\beta^{2}\right)^{2}$

Theorem 3.1. Let $F^{2}(x, y)=\frac{\beta^{2 n+2}}{\alpha^{2 n}}$ be the metric function of a Finsler space with generalized Kropina metric, for which the condition (2.7) is true, Then

$$
V_{j}^{i}=X_{k}^{i} Y_{j}^{k}
$$

is non holomic Finsler Frame with $X_{k}^{i}$ and $Y_{j}^{k}$ are given by (3.2) and (3.3) respectively.

Case I: Consider $n=1$ the case of nonholonomic Finlser frame with Kropina Metric $L(\alpha, \beta)=\frac{\beta^{4}}{\alpha^{2}}$

$$
\rho_{1}=-\frac{\beta^{4}}{\alpha^{4}} \rho_{0}=\frac{6 \beta^{2}}{\alpha^{2}}, \quad \rho_{-1}=-\frac{4 \beta^{3}}{\alpha^{4}}, \quad \rho_{-2}=\frac{4 \beta^{4}}{\alpha^{6}},
$$




$$
B^{2}=\frac{16 \beta^{6}\left(\alpha^{2} b^{2}-\beta^{2}\right)}{\alpha^{10}}
$$

Using (3.4) in (2.10) we have,

$$
\begin{array}{r}
X_{j}^{i}=\sqrt{-1} \frac{\beta^{2}}{\alpha^{2}} \delta_{j}^{i}-16 \frac{\beta^{4}}{\alpha^{8}}\left\{\sqrt{-1} \frac{\beta^{2}}{\alpha^{2}}+\sqrt{\left(-1 \frac{\beta^{4}}{\alpha^{4}}+\frac{\alpha^{6}}{4 \beta^{2}}\right)}\right\}\left(b^{i}-\right. \\
\left.\frac{\beta}{\alpha^{2}} y^{i}\right)\left(b_{j}-\frac{\beta}{\alpha^{2}} y_{j}\right)
\end{array}
$$

Again using (3.4) in (2.12) we have

$$
Y_{j}^{i}=\delta_{j}^{i}-\frac{1}{C^{2}}\left\{1 \pm \sqrt{1+\frac{\alpha^{2} C^{2}}{2 \beta^{2}}}\right\} b^{i} b_{j}
$$

where $C^{2}=-\frac{\beta^{4}}{\alpha^{4}} b^{2}+\frac{4 \beta^{2}}{\alpha^{6}}\left(\alpha^{2} b^{2}-\beta^{2}\right)^{2}$

Corollary 3.1. Let $F^{2}(x, y)=\frac{\beta^{4}}{\alpha^{2}}$ be the metric function of a Finsler space with special generalized Kropina metric, for which the condition (2.7) is true, Then

$$
V_{j}^{i}=X_{k}^{i} Y_{j}^{k}
$$

is non holomic Finsler Frame with $X_{k}^{i}$ and $Y_{j}^{k}$ are given by (3.5) and (3.6) respectively.

Case II: Consider $n=2$ the case of nonholonomic Finlser frame with Kropina Metric $L(\alpha, \beta)=\frac{\beta^{6}}{\alpha^{4}}$

$$
\begin{gathered}
\rho_{1}=-\frac{\beta^{6}}{\alpha^{6}} \rho_{0}=\frac{10 \beta^{4}}{\alpha^{4}}, \quad \rho_{-1}=-\frac{12 \beta^{5}}{\alpha^{6}}, \quad \rho_{-2}=\frac{8 \beta^{6}}{\alpha^{8}}, \\
B^{2}=\frac{144 \beta^{10}\left(\alpha^{2} b^{2}-\beta^{2}\right)}{\alpha^{14}}
\end{gathered}
$$

Using (3.7) in (2.10) we have,

$$
\begin{array}{r}
X_{j}^{i}=\sqrt{-2} \frac{\beta^{3}}{\alpha^{3}} \delta_{j}^{i}-144 \frac{\beta^{8}}{\alpha^{12}}\left\{\sqrt{-2} \frac{\beta^{3}}{\alpha^{3}}+\sqrt{\left(-2 \frac{\beta^{6}}{\alpha^{6}}+\frac{\alpha^{8}}{12 \beta^{4}}\right)}\right\}\left(b^{i}-\right. \\
\left.\frac{\beta}{\alpha^{2}} y^{i}\right)\left(b_{j}-\frac{\beta}{\alpha^{2}} y_{j}\right)
\end{array}
$$

Again using (3.7) in (2.12) we have,

$$
Y_{j}^{i}=\delta_{j}^{i}-\frac{1}{C^{2}}\left\{1 \pm \sqrt{1+\frac{\alpha^{4} C^{2}}{3 \beta^{4}}}\right\} b^{i} b_{j}
$$


where $C^{2}=-\frac{2 \beta^{6}}{\alpha^{6}} b^{2}+\frac{12 \beta^{4}}{\alpha^{8}}\left(\alpha^{2} b^{2}-\beta^{2}\right)^{2}$.

Corollary 3.2. Let $F^{2}(x, y)=\frac{\beta^{6}}{\alpha^{4}}$ be the metric function of a Finsler space with special generalized Kropina metric, for which the condition (2.7) is true, Then

$$
V_{j}^{i}=X_{k}^{i} Y_{j}^{k}
$$

is non holomic Finsler Frame with $X_{k}^{i}$ and $Y_{j}^{k}$ are given by (3.8) and (3.9) respectively.

\section{Conclusions}

Non-holonomic frame relates a semi-Riemannian metric (the Minkowski or the Lorentz metric) with an induced Finsler metric. Antonelli and Bucataru (see [7], [8]), has been determined such a non-holonomic frame for two important classes of Finsler spaces that are dual in the sense of Randers and Kropina spaces [9]. As Randers and Kropina spaces are members of a bigger class of Finsler spaces, namely the Finsler spaces with $(\alpha, \beta)$-metric, it appears a natural question: Does how many Finsler space with $(\alpha, \beta)$-metrics have such a nonholonomic frame? The answer is yes, there are many Finsler space with $(\alpha$, $\beta)$-metrics.

In this work, we consider the Generalized Kropina Finsler metrics and we determine the nonholonomic Finsler frames. Further we discuss two cases for $\mathrm{n}=1$ and $\mathrm{n}=2$ in Generalized Kropina Finsler metrics and we determine also the non holonomic Finsler frames for them.we found here induces a Finsler connection on TM with torsion and no curvature. But, in Finsler geometry, there are many $(\alpha, \beta)$-metrics, in future work we can determine the frames for them also.

\section{References}

[1] Holland, P.R.: Electromagnetism, Particles and Anholonomy. Physics Letters, 91(6) (1982), 275-278.

[2] Holland, P.R.: Anholonomic deformations in the ether: a significance for the electrodynamic potentials. In: Hiley, B.J. Peat, F.D. (eds.), Quantum Implications. Routledge and Kegan Paul, London and New York, 295-311 (1987).

[3] Ingarden, R.S.: On Physical interpretations of Finsler and Kawaguchi spaces. Tensor N.S., 46,(1987), 354-360. 
[4] Randers, G.: On asymmetric metric in the four space of general relativity. Phys. Rev., 59,(1941), 195-199.

[5] Beil, R.G.: Comparison of unified field theories. Tensor N.S., 56,(1995), 175-183.

[6] Beil, R.G.: Equations of Motion from Finsler Geometric Methods. In: Antonelli, P.L. (ed), Finslerian Geometries. A meeting of minds. Kluwer Academic Publisher, FTPH, no. 109, (2000), 95-111.

[7] Antonelli, P.L., Bucataru, I.: On Holland's frame for Randers space and its applications in physics. In: Kozma, L. (ed), Steps in Differential Geometry. Proceedings of the Colloquium on Differential Geometry, Debrecen, Hungary, July 25-30, 2000. Debrecen: Univ. Debrecen, Institute of Mathematics and Informatics, 39-54, (2001).

[8] Antonelli, P.L., Bucataru, I.: Finsler connections in anholonomic geometry of a Kropina space. Nonlinear Studies, 8 (1), (2001), 171-184.

[9] Hrimiuc, D., Shimada, H.: On the L-duality between Lagrange and Hamilton manifolds. Nonlinear World, 3, (1996), 613-641.

[10] Ioan Bucataru, Radu Miron: Finsler-Lagrange Geometry. Applications to dynamical systems CEEX ET 3174/2005-2007, and CEEX M III 12595/2007 (2007).

[11] Matsumoto, M.: Theory of Finsler spaces with $(\alpha, \beta)$-metric, Rep. Math. Phys., 31, (1992), 43-83.

[12] Bucataru, I.: Nonholonomic frames on Finsler geometry. Balkan Journal of Geometry and its Applications, 7 (1), (2002), 13-27.

[13] Matsumoto. M: Foundations of Finsler geometry and special Finsler spaces, Kaishesha Press, Otsu, Japan, 1986.

[14] Tripathi Brijesh Kumar,Pandey K. B. and Tiwari R.B.:Nonholonomic Frame for Finsler Space with $(\alpha, \beta)$-metric,International J. Math. Combin. Vol.1, (2016), 109-115.

[15] Narasimhamurthy S.K., Mallikarjun Y. Kumar,Kavyashree A. R.: Nonholonomic Frames For Finsler Space With Special $(\alpha, \beta)$-metric, International Journal of Scientific and Research Publications,4(1), (2014), 1-7. 